\title{
Compressibilidade do solo e sistema radicular da cana-de-açúcar em manejo com e sem controle de tráfego
}

\author{
Gustavo Soares de Souza(1), Zigomar Menezes de Souza(1), Reginaldo Barboza da Silva(2), \\ Fernando Silva Araújo(1) e Ronny Sobreira Barbosa ${ }^{(1)}$
}

\begin{abstract}
(1) Universidade Estadual de Campinas, Faculdade de Engenharia Agrícola, Avenida Candido Rondon, no 501, Barão Geraldo, CEP 13083-875 Campinas, SP. E-mail: gustavo.souza@feagri.unicamp.br, zigomarms@feagri.unicamp.br (2)Universidade Estadual Paulista, Campus de Registro, Rua Nelson Brihi Badur, no 430, CEP 11900-000 Registro, SP. E-mail: rbsilva@registro.unesp.br
\end{abstract}

Resumo - O objetivo deste trabalho foi comparar a capacidade de suporte de carga do solo em área com cana-de-açúcar colhida mecanicamente sem queima, em sistemas de manejo com e sem controle de tráfego agrícola. O controle de tráfego foi feito com ajuste da bitola do trator e transbordo, ou com ajuste da bitola e uso de piloto automático. As amostras de solo foram coletadas em cilindros volumétricos, na soqueira e na entrelinha da cultura (linha de rodado), nas camadas de $0,00-0,10$ e $0,20-0,30 \mathrm{~m}$. Avaliou-se a densidade radicular por meio de imagens, obtidas da digitalização de raízes coletadas em monólitos de $0,25 \times 0,10 \times 0,10 \mathrm{~m}$. O manejo sem controle de tráfego apresentou maior capacidade de suporte de carga do solo na linha de plantio, nas duas camadas de solo avaliadas, o que indicou maior compactação. Maior densidade radicular ocorreu no manejo com controle de tráfego com ajuste da bitola e piloto automático, que permitiu maior capacidade de suporte de carga na linha de rodado e preservou a qualidade estrutural na região da soqueira, com reflexo positivo sobre o desenvolvimento do sistema radicular da cana-de-açúcar.

Termos para indexação: Saccharum, capacidade de suporte de carga, colheita mecanizada, estrutura do solo, pressão de preconsolidação.

\section{Soil compressibility and root system of sugarcane with and without controlled-traffic farming}

\begin{abstract}
The objective of this work was to compare the load-carrying capacity of the soil in a mechanically harvested sugarcane area, without burning, in managements with and without controlled traffic farming. Controlled traffic was done adjusting the gauges of tractor and trailer, or adjusting the gauges and using autopilot. Soil samples were collected in volumetric cylinders in plant rows and inter-rows (wheel rows), from $0.00-0.10$ and $0.20-0.30-\mathrm{m}$ soil depths. Root density was measured by images, obtained by scanning the collected roots in $0.25 \times 0.10 \times 0.10$-m monoliths. The management without controlled traffic showed a higher load-carrying capacity of the soil in the planting rows, in both soil layers, which indicates a higher compaction. Greater root density occurred in the management with controlled traffic with gauge adjustment and use of autopilot, which made possible a higher load-carrying capacity in wheel rows, and preserved structural quality in the plant rows, resulting in a greater root system development of sugarcane.
\end{abstract}

Index terms: Saccharum, load-carrying capacity, mechanical harvest, soil structure, preconsolidation pressure.

\section{Introdução}

O intenso número de operações mecanizadas em lavouras de cana-de-açúcar resulta na compactação do solo, com efeitos danosos sobre sua qualidade física. Atualmente, tem-se observado aumento de áreas agrícolas com problemas de compactação, em grande parte atribuído a operações mecanizadas, realizadas sem se considerar a umidade do solo (Saffih-Hdadi et al., 2009). A compactação resulta em problemas ambientais, agronômicos e econômicos como inundação, erosão, lixiviação de agrotóxicos, emissão de gases de efeito estufa e perda de rendimento das culturas agrícolas (Keller \& Lamandé, 2010).
Pesquisas têm mostrado a relação entre compactação e qualidade física do solo. A compactação aumenta a densidade, a resistência mecânica à penetração e a pressão de preconsolidação dos solos e diminui seu volume de poros (Braunack \& McGarry, 2006; Braunack et al., 2006; Silva et al., 2009). Para o desenvolvimento da planta, é fundamental que o solo apresente condições favoráveis ao crescimento das raízes, para que a planta possa explorar maior volume em camadas mais profundas, e ter maior acesso à água, o que reduziria os riscos de deficiência hídrica (Braunack et al., 2006; Collares et al., 2008; Kaiser et al., 2009). 
Uma alternativa atual para o problema de compactação do solo sob canaviais é o manejo com controle de tráfego agrícola (Roque et al., 2010), que preserva, nas regiões não trafegadas, as propriedades do solo e favorece o desenvolvimento do sistema radicular e o desenvolvimento das culturas (Braunack \& McGarry, 2006; Vermeulen \& Mosquera, 2009). O controle de tráfego separa zonas de tráfego de zonas de crescimento das plantas, e concentra a passagem de pneus em linhas permanentes, com diminuição da área submetida ao tráfego agrícola (Braunack et al., 2006; Roque et al., 2010; Kingwell \& Fuchsbichler, 2011). Essa técnica tem promovido melhorias na qualidade estrutural dos solos agrícolas e maior desenvolvimento das culturas (Braunack et al., 2006). O uso de espaçamentos coincidentes entre todos os maquinários agrícolas, por meio do ajuste da bitola, melhora, ainda, as condições de tráfego em solo com maior teor de água e aumenta a eficiência de tração (Kingwell \& Fuchsbichler, 2011).

Em complemento ao controle de tráfego, tem sido utilizado o sistema de direção assistida (piloto automático). Este equipamento é utilizado para guiar as máquinas agrícolas em operações de campo, de tal forma que o deslocamento ocorra sempre paralelo a uma linha de referência, o que resulta na maior uniformização do espaçamento, com melhor aproveitamento do terreno e menor tráfego sobre as linhas de cultivo (Vermeulen \& Mosquera, 2009).

Uma medida efetiva na prevenção da compactação do solo é a aplicação de pressões inferiores à capacidade de suporte de carga, definida como a capacidade de uma estrutura de solo em resistir a tensões induzidas pelo tráfego de máquinas no campo, sem mudanças no arranjo tridimensional das partículas do solo (Debiasi et al., 2008). A capacidade de suporte de carga do solo relaciona a pressão de preconsolidação à umidade do solo, já que esta reduz a resistência interna do solo, o que o torna mais suscetível ao processo compressivo (Imhoff et al., 2004; Suzuki et al., 2008).

Indicações na literatura ressaltam a importância de se caracterizar o processo compressivo dos solos para prevenir sua degradação física (Keller et al., 2004; Lima et al., 2006; Suzuki et al., 2008; Silva et al., 2009). A aplicação de pressões maiores do que a capacidade de suporte de carga no solo causa deformações não recuperáveis, o que resulta na degradação da estrutura do solo (Imhoff et al., 2004; Silva et al., 2009).
Além disso, solos com valores elevados de pressão de preconsolidação apresentam maior resistência mecânica à penetração de raízes (Pacheco \& Cantalice, 2011), o que é deletério ao crescimento e produtividade das culturas (Lima et al., 2006). Quantificar os níveis de pressão suportados pelo solo contribui para o desenvolvimento sustentável do setor sucroalcooleiro, permite evitar a degradação estrutural dos solos e adaptar as atividades de forma condizente com as limitações dos ambientes de produção.

A aplicação do controle de tráfego em canaviais pode aumentar a capacidade de suporte de carga do solo na linha de rodado, melhorar a interação solo-máquina e preservar a qualidade estrutural na região da soqueira.

O objetivo deste trabalho foi comparar a capacidade de suporte de carga do solo, em área com cana-de-açúcar colhida mecanicamente e sem queima, em sistema de manejo com e sem controle de tráfego agrícola.

\section{Material e Métodos}

A pesquisa foi realizada na safra agrícola 2010/2011, em lavoura comercial no Município de Pradópolis, SP $\left(21^{\circ} 18^{\prime} 67^{\prime \prime} \mathrm{S}, 48^{\circ} 11^{\prime} 38^{\prime \prime} \mathrm{W}\right.$, a $630 \mathrm{~m}$ de altitude). O clima é mesotérmico de inverno seco (Cwa), pela classificação de Köppen, com precipitação média de $1.400 \mathrm{~mm}$ e chuvas concentradas no período de novembro a fevereiro.

O ensaio foi conduzido em talhão de cana-de-açúcar (Saccharum sp.), cultivar RB855453, com 16,2 ha. A cultura foi instalada em agosto de 2007 e, em 2010, apresentou a produtividade referente à terceira colheita (segunda soca). O solo, de acordo com Santos et al. (2006), é um Latossolo Vermelho distrófico típico álico, de textura argilosa, com horizonte A moderado e relevo suave-ondulado. O talhão apresenta histórico com mais de 30 anos consecutivos de cultivo intensivo de cana e, nos últimos 12 anos, a colheita foi mecanizada e sem queima.

O preparo da área, realizado em julho de 2007, foi composto, inicialmente, pela eliminação da soqueira do cultivo anterior e subsolagem à profundidade de $0,45 \mathrm{~m}$ apenas nos sulcos de plantio. Foram aplicados 2,5 $\mathrm{Mg} \mathrm{ha}^{-1}$ de calcário dolomítico, antes do revolvimento do solo com grade aradora, e $20 \mathrm{Mg} \mathrm{ha}^{-1}$ de torta de filtro, antes do plantio. Em julho de 2009 e 2010, após a colheita, 280 e $260 \mathrm{~kg} \mathrm{ha}^{-1}$ do formulado

Pesq. agropec. bras., Brasília, v.47, n.4, p.603-612, abr. 2012 
32-00-02 foram aplicados, além de $100 \mathrm{~m}^{3} \mathrm{ha}^{-1}$ de vinhaça.

Nas operações mecanizadas, foi usado um trator Case MX-270, com tração 4x4, potência máxima do motor de $270 \mathrm{cv}(198 \mathrm{~kW})$, massa de $11,7 \mathrm{Mg}$ e pneus traseiros 650-85 R38 e dianteiros 600-70 R30, com pressão de inflação de 110 e $150 \mathrm{kPa}$, respectivamente, para tracionar os implementos. Na colheita realizada em junho de 2010, foi usada a colhedora de esteira Case A-7700, com potência máxima do motor de $335 \mathrm{cv}$ $(246 \mathrm{~kW})$ e massa de $18,5 \mathrm{Mg}$, e o trator Case MX-270, que arrastava um transbordo de três compartimentos, com massa total média de $40 \mathrm{Mg}$ e pneus 600-50 R22,5, com pressão de inflação de $110 \mathrm{kPa}$.

Os sistemas de manejo instalados em lavoura comercial de cana-de-açúcar, com colheita mecanizada e sem queima, foram: testemunha $(\mathrm{T})$, ausência de controle de tráfego na lavoura, com espaçamento entre linhas de cultivo de $1,5 \mathrm{~m}$ e bitola do trator e transbordo de 2,0 m; controle de tráfego (CT1), com espaçamento entre linhas de $1,5 \mathrm{~m}$ e ajuste da bitola do trator e transbordo para $3,0 \mathrm{~m}$; e controle de tráfego (CT2) com espaçamento entre linhas de 1,5 m, ajuste da bitola do trator e transbordo para 3,0 m e uso de piloto automático no plantio e nas colheitas subsequentes. A fundamentação para proposição desses sistemas de manejo considerou o conceito de "canteiro da cana"; neste caso, uma área de pelo menos $0,40 \mathrm{~m}$, de cada lado da linha de cultivo, não recebe tráfego de rodados do conjunto trator-transbordo.

As avaliações, em cada sistema de manejo, foram realizadas em amostras de solo com estrutura preservada, retiradas com cilindros volumétricos de $0,0635 \mathrm{~m}$ de diâmetro por $0,0254 \mathrm{~m}$ de altura, coletadas da linha de plantio e do centro da entrelinha de plantio (linha do rodado), no centro das camadas de $0,00-0,10$ e 0,20-0,30 m, para determinação de atributos físicos e da pressão de preconsolidação. Amostras deformadas $(n=4)$ foram usadas na caracterização da consistência do solo, definida pelos limites de contração de $0,20 \mathrm{~kg} \mathrm{~kg}^{-1}$ (Bowles, 1986), de plasticidade de $0,34 \mathrm{~kg} \mathrm{~kg}^{-1}$, e liquidez de $0,42 \mathrm{~kg} \mathrm{~kg}^{-1}$ (Sowers, 1965). Esses limites definem as faixas de tenacidade $\left(<0,20 \mathrm{~kg} \mathrm{~kg}^{-1}\right)$, friabilidade $\left(0,20-0,34 \mathrm{~kg} \mathrm{~kg}^{-1}\right) \mathrm{e}$ plasticidade $\left(0,34-0,42 \mathrm{~kg} \mathrm{~kg}^{-1}\right)$ do solo.

A capacidade de suporte de carga do solo representa uma função que relaciona a pressão de preconsolidação do solo com a umidade gravimétrica. As amostras de estrutura preservada foram saturadas e depois secas ao ar, no laboratório, para obtenção de amostras na faixa de umidade com variação de solo seco a saturado, tendo-se simulado as condições extremas de umidade do campo (Dias Júnior, 1994; Silva et al., 2009). A pressão de preconsolidação foi quantificada por meio do ensaio de compressão uniaxial, tendo-se aplicado cargas de $25,50,100,200,400,800$ e $1.600 \mathrm{kPa}$ às amostras indeformadas. Cada pressão foi aplicada até atingir $90 \%$ da deformação máxima e, em seguida, foi aumentada. Os ensaios de compressão uniaxial foram realizados em consolidômetro automático, com interação entre homem e máquina, modelo CNTA-IHM/ BR-001/07, acoplado ao programa específico que acompanha o equipamento.

Após o ensaio de compressão, as amostras foram secas em estufa a $105^{\circ} \mathrm{C}$, até obtenção de massa constante, para determinação da densidade do solo (Claessen, 1997). A partir da curva de compressão (densidade do solo $\mathrm{x}$ pressão), foi estimada a pressão de preconsolidação, conforme Dias Júnior (1994). Curvas de compressão do solo foram obtidas para as amostras em diferentes teores de umidade, o que permitiu a construção do modelo de capacidade de suporte do solo. Os dados experimentais foram ajustados à equação: $\sigma \mathrm{p}=10^{(\mathrm{a}+\mathrm{b} \times \mathrm{U})}$ (Dias Júnior, 1994), em que $\sigma p$ é a pressão de preconsolidação do solo, a e b são coeficientes da equação, e U é a umidade gravimétrica do solo.

Empregou-se o delineamento experimental de blocos ao acaso, com três sistemas de manejo e quatro blocos, com 12 parcelas de 14 linhas de cultivo de $50 \mathrm{~m}$ de comprimento. Cada parcela tinha duas subdivisões: locais de amostragem e camadas de solo. A capacidade de suporte do solo foi determinada nos três manejos, em dois locais de amostragem (linhas do rodado e de plantio) e em duas camadas de solo $(0,00-0,10$ e $0,20-0,30 \mathrm{~m})$. Para caracterizar o modelo de capacidade de suporte de carga do solo, foram amostrados 20 cilindros para cada tratamento, no total de 240 amostras. As equações foram ajustadas para cada tratamento e comparadas de acordo com Snedecor \& Cochran (1989).

O sistema radicular foi avaliado pelo método do monólito (Vasconcelos et al., 2003; Faroni \& Trivelin, 2006). Os monólitos, com dimensão de $0,25 \times 0,10 \times 0,10 \mathrm{~m}$ para a largura, altura e comprimento, respectivamente, foram coletados em junho de 2010, logo após a colheita, em quatro trincheiras (blocos), nos diferentes sistemas de manejo. Em cada trincheira, 
foram coletados 18 monólitos, de forma perpendicular, entre duas linhas de cana, nas camadas de $0,00-0,10$; $0,10-0,20$ e $0,20-0,30 \mathrm{~m}$, no total de 216 amostras.

As raízes foram separadas do solo por lavagem em água corrente, com uso de peneira de 2,0 mm (Vasconcelos et al., 2003; Faroni \& Trivelin, 2006), e suas imagens foram digitalizadas em scanner de leitura ótica, com resolução de 400 dpi, para a análise da densidade radicular com o programa Siarcs (Jorge \& Crestana, 1996). Os dados médios de cada sistema de manejo foram interpolados, o que permitiu a espacialização da densidade radicular na região entre duas soqueiras.

\section{Resultados e Discussão}

O controle de tráfego proporcionou menores valores de densidade do solo e maiores de porosidade na linha de plantio (soqueira), nas duas camadas em estudo, em comparação ao manejo sem controle de tráfego (Tabela 1). Isso ocorreu em razão da ausência do tráfego de pneus do conjunto motomecanizado trator-transbordo sobre, ou próximo, à soqueira, no manejo com controle de tráfego, em consequência do ajuste da bitola e uso do piloto automático. O controle de tráfego é uma prática recente, que tem como objetivo preservar as condições ideais para o crescimento das culturas nos locais não trafegados (Vermeulen \& Mosquera, 2009; Kingwell \& Fuchsbichler, 2011).

Sob a linha de rodado, o solo apresentou maior densidade e menor porosidade total no manejo com controle de tráfego, em ambas as camadas (Tabela 1). A compactação na linha de rodado é intensificada

Tabela 1. Atributos físicos do solo $(n=20)$ em manejos com controle de tráfego, por meio de ajuste da bitola e uso de piloto automático (CT2), e por meio do ajuste da bitola apenas (CT1); e em testemunha sem controle de tráfego (T). Os atributos foram determinados nas linhas de plantio e de rodado, nas camadas de $0,00-0,10$ e $0,20-0,30 \mathrm{~m}$.

\begin{tabular}{llllll}
\hline Sistema de manejo & \multicolumn{2}{c}{ Linha de rodado } & & \multicolumn{3}{c}{ Linha de plantio } \\
\cline { 2 - 3 } \cline { 5 - 6 } & $0,00-0,10 \mathrm{~m}$ & $0,20-0,30 \mathrm{~m}$ & & $0,00-0,10 \mathrm{~m}$ & $0,20-0,30 \mathrm{~m}$ \\
\hline \multirow{5}{c}{ Densidade do solo $\left(\mathrm{Mg} \mathrm{m}^{-3}\right)$} \\
CT2 & $1,46 \pm 0,09$ & $1,31 \pm 0,07$ & & $1,19 \pm 0,07$ & $1,21 \pm 0,10$ \\
CT1 & $1,35 \pm 0,10$ & $1,29 \pm 0,10$ & & $1,13 \pm 0,11$ & $1,16 \pm 0,13$ \\
$\mathrm{~T}$ & $1,30 \pm 0,09$ & $1,29 \pm 0,07$ & $1,26 \pm 0,14$ & $1,24 \pm 0,07$ \\
\hline \multirow{5}{c}{ Porosidade total $\left(\mathrm{m}^{3} \mathrm{~m}^{-3}\right)$} \\
CT2 & $0,48 \pm 0,03$ & $0,53 \pm 0,03$ & $0,58 \pm 0,03$ & $0,57 \pm 0,04$ \\
CT1 & $0,52 \pm 0,04$ & $0,54 \pm 0,04$ & $0,60 \pm 0,04$ & $0,58 \pm 0,05$ \\
T & $0,54 \pm 0,03$ & $0,54 \pm 0,02$ & $0,55 \pm 0,05$ & $0,56 \pm 0,03$ \\
\hline
\end{tabular}

com o controle de tráfego, já que este é concentrado em linhas permanentes (Braunack et al., 2006; Roque et al., 2010; Kingwell \& Fuchsbichler, 2011). O uso do piloto automático e o ajuste da bitola do conjunto trator-transbordo contribuíram para concentrar $\mathrm{o}$ tráfego na linha de rodado, o que resultou na maior densidade do solo, em comparação ao manejo apenas com o ajuste da bitola. Assim, o sistema de manejo com controle de tráfego alia uma melhor condição de tráfego na linha do rodado, com a ausência de tráfego na linha de plantio, o que favorece o desenvolvimento das culturas agrícolas (Braunack \& McGarry, 2006).

O índice de compressão e a deformação do solo, medidos nos corpos-de-prova durante os ensaios de compressibilidade, apresentaram relação direta com a densidade e a umidade do solo. Incrementos no teor de água do solo aumentaram a deformação e o índice de compressão do solo $\left(\mathrm{r}=0,74^{* *}\right.$ e $\left.0,70^{* *}\right)$. Imhoff et al. (2004) e Saffih-Hdadi et al. (2009) também observaram redução do índice de compressão com o aumento da umidade. Isso ocorre porque a água atua como lubrificante no solo e reduz o atrito interno entre as partículas e a resistência dos agregados à ruptura, permitindo maior acomodação das partículas do solo no processo de compressibilidade (Lima et al., 2006; Silva et al., 2009).

O aumento da densidade do solo reduziu a deformação e o índice de compressão $(\mathrm{r}=-0,59 * *$ e $\left.-0,57^{* *}\right)$. Esses resultados concordam com os de Imhoff et al. (2004) e Suzuki et al. (2008). Maiores densidades aumentam o atrito interno entre partículas sólidas, em consequência do maior número de pontos de contato, e reduzem a compressibilidade do solo (Pacheco \& Cantalice, 2011). Conforme Suzuki et al. (2008), o aumento da densidade promove redução dos vazios do solo e aumento do grau de compactação, o que contribui para reduzir a deformação e o índice de compressão. Isso contribuiu para que a suscetibilidade do solo à compactação seja diminuída, aumentando sua capacidade de suporte de carga e favorecendo o tráfego de máquinas; contudo, elevadas densidades também podem limitar o crescimento radicular das plantas (Lima et al., 2006; Suzuki et al., 2008).

O aumento da umidade do solo reduziu a pressão de preconsolidação do solo, representado nos modelos de capacidade de suporte de carga $\left[\sigma p=10^{(\mathrm{a}+\mathrm{b} \times \mathrm{U})}, \mathrm{n}=20\right]$ (Figura 1). O aumento do teor de água no solo diminui sua resistência interna, em consequência da redução

Pesq. agropec. bras., Brasília, v.47, n.4, p.603-612, abr. 2012 
da coesão entre as partículas sólidas, e forma uma película sobre estas e os microagregados que reduz o atrito entre elas (Pacheco \& Cantalice, 2011). Se os fatores do solo se mantêm constantes, a umidade do solo governa a magnitude da deformação (Lima et al., 2006; Saffih-Hdadi et al., 2009).

Os sistemas de manejo com controle de tráfego, no presente trabalho, interferiram significativamente nos modelos de capacidade de suporte de carga do solo, com base no teste F de homogeneidade de dados de Snedecor \& Cochran (1989), com exceção para os dados da linha de plantio na camada $0,00-0,10 \mathrm{~m}$ (Tabela 2). Pacheco \& Cantalice (2011) também observaram que diferentes tipos de cultivo e histórico de uso da cana-de-açúcar alteraram a compressibilidade do solo.

Como não houve diferença estatística entre os sistemas de manejo com controle de tráfego, optouse por ajustar um modelo único com seus dados de pressão de preconsolidação e umidade, e compará-lo ao manejo sem controle de tráfego (Figura 2). Diferenças significativas ocorreram entre os sistemas de manejo com e sem controle de tráfego, nos locais de amostragem e camadas em estudo.

Para qualquer valor de umidade do solo, o manejo sem controle de tráfego apresentou maior pressão de preconsolidação do solo na linha de plantio, nas duas camadas de solo (Figura 2). Considerando-se que o tráfego de máquinas deve ser realizado em condições de umidade inferior à do limite de plasticidade do solo (Silva et al., 2009), que, no presente estudo, foi menor do que $0,34 \mathrm{~kg} \mathrm{~kg}^{-1}$, na região de friabilidade ou tenacidade, observou-se maior capacidade de suporte de carga do solo no manejo sem controle de tráfego, nas duas camadas de solo (315 e $258 \mathrm{kPa}$ ), em comparação ao manejo com controle de tráfego (281 e $223 \mathrm{kPa})$. Isso é caracterizado pelo efeito do tráfego de pneus, do conjunto trator-transbordo próximo à soqueira da cultura da cana-de-açúcar, ao longo dos subsequentes

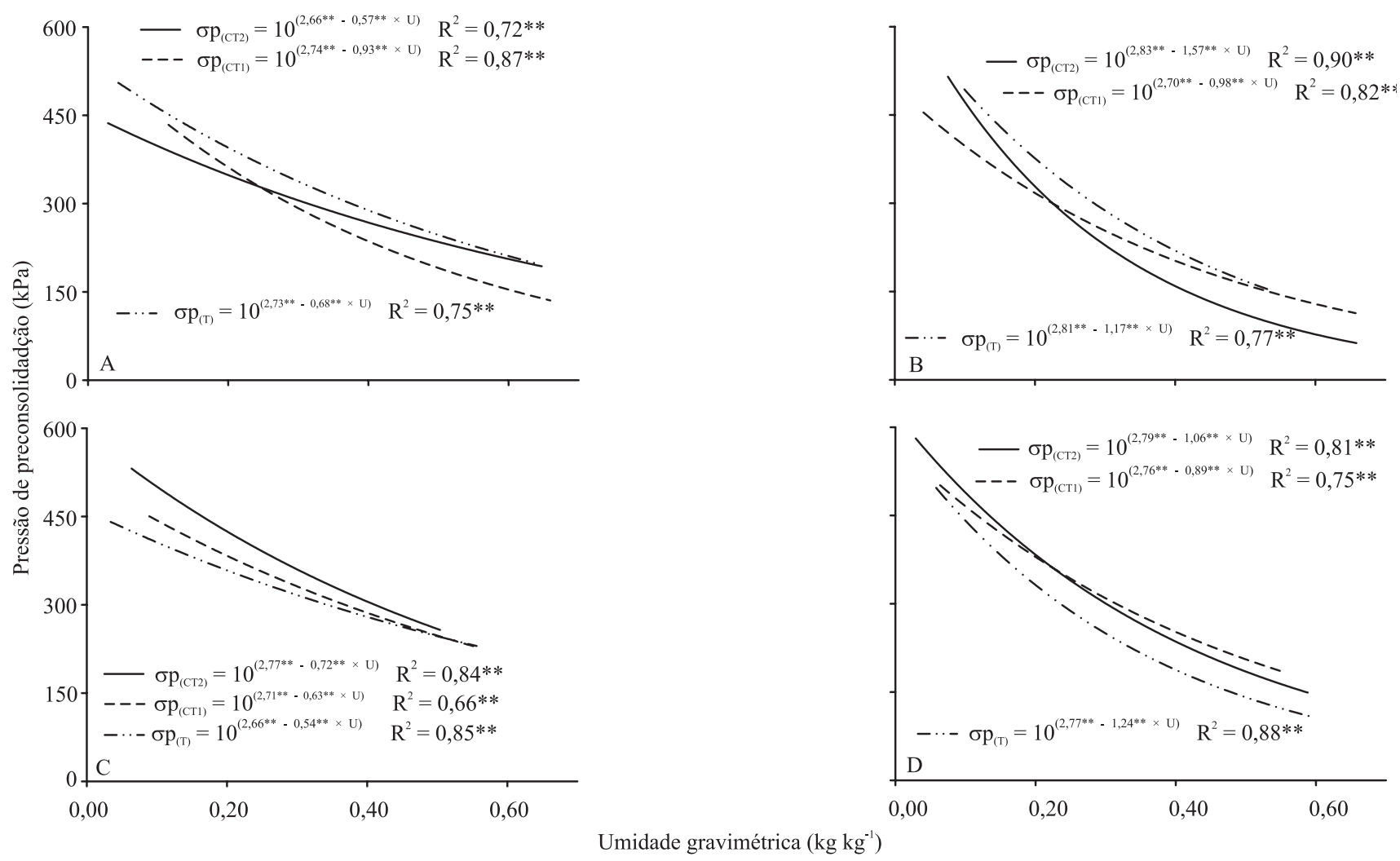

Figura 1. Modelos de capacidade de suporte de carga do solo $(n=20)$, para os seguintes sistemas de manejo: controle de tráfego por meio do ajuste da bitola e uso de piloto automático (CT2), e por meio do ajuste da bitola apenas (CT1); e testemunha sem controle de tráfego $(\mathrm{T})$. Os modelos foram determinados nas linhas de plantio (LP) e de rodado (LR), nas camadas de 0,00-0,10 e 0,20-0,30 m. A, LP 0,010-0,10 m; B, LP 0,20-0,30 m; C, LR 0,00-0,10 m; D, LR 0,20-0,30 m. $\sigma p$, pressão de preconsolidação. 
ciclos da cultura, que tornou o solo mais compactado e mais resistente a deformações.

No manejo com controle de tráfego em cana-de-açúcar não se utiliza o tráfego de pneus do conjunto trator-transbordo sobre ou próximo à linha da soqueira, para evitar danos ao volume total de poros e, por conseguinte, à estrutura do solo (Tabela 1, Figura 2) próximo às plantas. Braunack et al. (2006) e Braunack \& McGarry (2006) observaram melhorias na qualidade física do solo na região da soqueira, com emprego de sistema de manejo com controle de tráfego agrícola, em área cultivada com cana-de-açúcar na Austrália, em comparação ao manejo sem controle de tráfego. Roque et al. (2010), no entanto, não observaram mudanças na qualidade física do solo na região das soqueiras, após dois anos de controle de tráfego na mesma área de cultivo de cana-de-açúcar deste trabalho.

O solo com controle de tráfego apresentou maior pressão de preconsolidação, para qualquer umidade do solo, na linha do rodado, nas duas camadas em

Tabela 2. Teste de significância, na comparação dos modelos de capacidade de suporte de carga do solo, de manejos com controle de tráfego por meio do ajuste da bitola e uso de piloto automático (CT2), e por meio do ajuste da bitola apenas (CT1); e testemunha sem controle de tráfego (T), nas linhas de plantio (LP) e de rodado (LR), nas camadas de $0,00-0,10$ e $0,20-0,30 \mathrm{~m}$.

\begin{tabular}{|c|c|c|c|c|}
\hline \multirow[t]{2}{*}{ Tratamento } & \multirow[t]{2}{*}{ Local e camada amostrados } & \multicolumn{3}{|c|}{ Estatística $^{(1)}$} \\
\hline & & $\mathrm{F}$ & $\mathrm{b}$ & $\mathrm{a}$ \\
\hline \multirow{5}{*}{ CT2 2 T } & \multicolumn{4}{|c|}{ Comparação dos sistemas de manejo } \\
\hline & LP $0,00-0,10$ & ns & ns & ns \\
\hline & LP $0,20-0,30$ & ns & ns & $*$ \\
\hline & LR $0,00-0,10$ & ns & $*$ & $* *$ \\
\hline & LR $0,20-0,30$ & ns & ns & $* *$ \\
\hline \multirow{3}{*}{$\mathrm{CT} 1 \times \mathrm{T}$} & LP $0,00-0,10$ & ns & ns & ns \\
\hline & LP $0,20-0,30$ & $*$ & ns & $*$ \\
\hline & LR $0,00-0,10$ & $* *$ & ns & ns \\
\hline \multirow{5}{*}{$\mathrm{CT} 1+\mathrm{CT} 2 \times \mathrm{T}$} & LR $0,20-0,30$ & ns & ns & $* *$ \\
\hline & LP $0,00-0,10$ & ns & ns & $*$ \\
\hline & LP $0,20-0,30$ & ns & ns & $*$ \\
\hline & LR $0,00-0,10$ & ns & $* *$ & $* *$ \\
\hline & LR $0,20-0,30$ & ns & ns & $* *$ \\
\hline \multirow{3}{*}{ LR $x$ LP } & \multicolumn{4}{|c|}{ Comparação dos locais de amostragem } \\
\hline & CT2 $0,00-0,10$ & $* *$ & ns & $* *$ \\
\hline & CT2 $0,20-0,30$ & ns & ns & $* *$ \\
\hline \multirow[t]{2}{*}{ LR x LP } & CT1 $0,00-0,10$ & ns & ns & $*$ \\
\hline & CT1 $0,20-0,30$ & ns & ns & $*$ \\
\hline \multirow[t]{2}{*}{ LR $x$ LP } & Т $0,00-0,10$ & ns & ns & ns \\
\hline & Т $0,20-0,30$ & ns & ns & $*$ \\
\hline
\end{tabular}

${ }^{(1)}$ Teste F de Snedecor \& Cochran (1989); b, coeficiente angular; a,

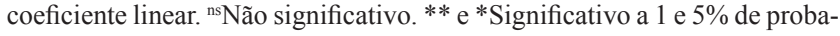
bilidade, respectivamente. Dados transformados: $\mathrm{x}^{\prime}=\log \mathrm{x}$. estudo (Figura 2). Considerando-se a umidade do solo referente ao limite de plasticidade, a capacidade de suporte de carga, no manejo com controle de tráfego, nas camadas de $0,00-0,10$ e 0,20-0,30 m, foi, respectivamente, de 323 e 280 $\mathrm{kPa}$, enquanto no manejo sem controle de tráfego foi de 300 e $223 \mathrm{kPa}$. Os resultados refletem o efeito da maior compactação do solo na linha do rodado (região central da entrelinha da cultura), no manejo com controle de tráfego, causada pelo tráfego de máquinas agrícolas, principalmente durante a colheita da cana, intensificado pela sobreposição das passadas dos pneus do conjunto trator-transbordo (Braunack et al., 2006). Contudo, deve-se observar até que ponto o solo pode suportar tal pressão, sem prejuízo à sua estrutura e, principalmente, ao desenvolvimento das plantas (Suzuki et al., 2008).

Um possível problema ao uso do controle de tráfego agrícola nos canaviais brasileiros, e presente neste trabalho, é o uso da bitola da colhedora de cana de $1,88 \mathrm{~m}$, que promove o tráfego da esteira próximo à soqueira. A colhedora de cana-de-açúcar confere uma pressão de contato estática (PCe) de $132 \mathrm{kPa}$ sobre a superfície do solo, se for considerada uma distribuição homogênea da carga ao longo da esteira. Essa PCe é inferior à pressão de preconsolidação do solo na camada superficial, com qualquer índice de umidade, na região da soqueira, o que indica adequação da máquina à preservação da estrutura do solo (Figura 2 A). Esse resultado indica elevada resistência interna entre partículas e agregados, que imprimiu maior estabilidade estrutural ao solo. Um dos principais responsáveis pela compactação nos canaviais tem sido o tráfego do conjunto trator-transbordo, na colheita, e de máquinas autopropelidas na aplicação de insumos, pois estas apresentam pequena área de contato pneu-solo, ao exercer pressões na superfície do solo que podem ultrapassar $450 \mathrm{kPa}$ (Silva et al., 2006).

Do ponto de vista prático e sustentável à estrutura do solo, o aumento na área de contato do rodado com o solo é eficiente em reduzir o risco de compactação causado pelo tráfego de máquinas (Keller \& Arvidsson, 2004; Silva et al., 2009). Outra medida preventiva tem sido o monitoramento da umidade do solo, e tentativa de realização das atividades mecanizadas em condição de umidade inferior à limitante, indicada no modelo de capacidade de suporte de carga. Por exemplo, um trator utilizado em operações agrícolas exerce uma 
pressão estática na superfície do solo de $363 \mathrm{kPa}$ (Silva et al., 2006), assim essa máquina deve trafegar no solo em condição de umidade inferior a $0,26 \mathrm{~kg} \mathrm{~kg}^{-1}$, para o manejo com controle de tráfego (Figura $2 \mathrm{C}$ ). Para o manejo sem controle de tráfego, a mesma pressão deve ser exercida, em condição de umidade inferior a $0,19 \mathrm{~kg} \mathrm{~kg}^{-1}$ (Figura $2 \mathrm{C}$ ). Assim, o manejo com ajuste da bitola e uso de piloto automático favorece os cronogramas de trabalho em empresas agrícolas, em razão da menor dependência da umidade do solo (que é influenciada pelas condições climáticas), pois permite o trabalho das máquinas agrícolas em solo mais úmido.

No manejo com controle de tráfego, ocorreu maior capacidade de suporte de carga do solo, na linha do rodado em comparação à linha de plantio, nas duas camadas de solo, evidenciada pelo maior coeficiente linear e menor coeficiente angular (Tabela 2). Esses resultados reforçam a ideia de que o manejo do canavial, com ajuste da bitola das máquinas e uso do piloto automático, preserva a qualidade física do solo na região da soqueira (Braunack \& McGarry, 2006; Kingwell \& Fuchsbichler, 2011).

Observou-se maior densidade radicular com o uso do controle de tráfego com ajuste da bitola e piloto automático, seguido do uso apenas do ajuste da bitola e do manejo sem controle de tráfego (Figura 3). Esses resultados expressam os benefícios do sistema de manejo com controle de tráfego para o cultivo da cana-de-açúcar. Braunack et al. (2006) estudaram o efeito do controle de tráfego, no cultivo da cana-de-açúcar, e verificaram a redução

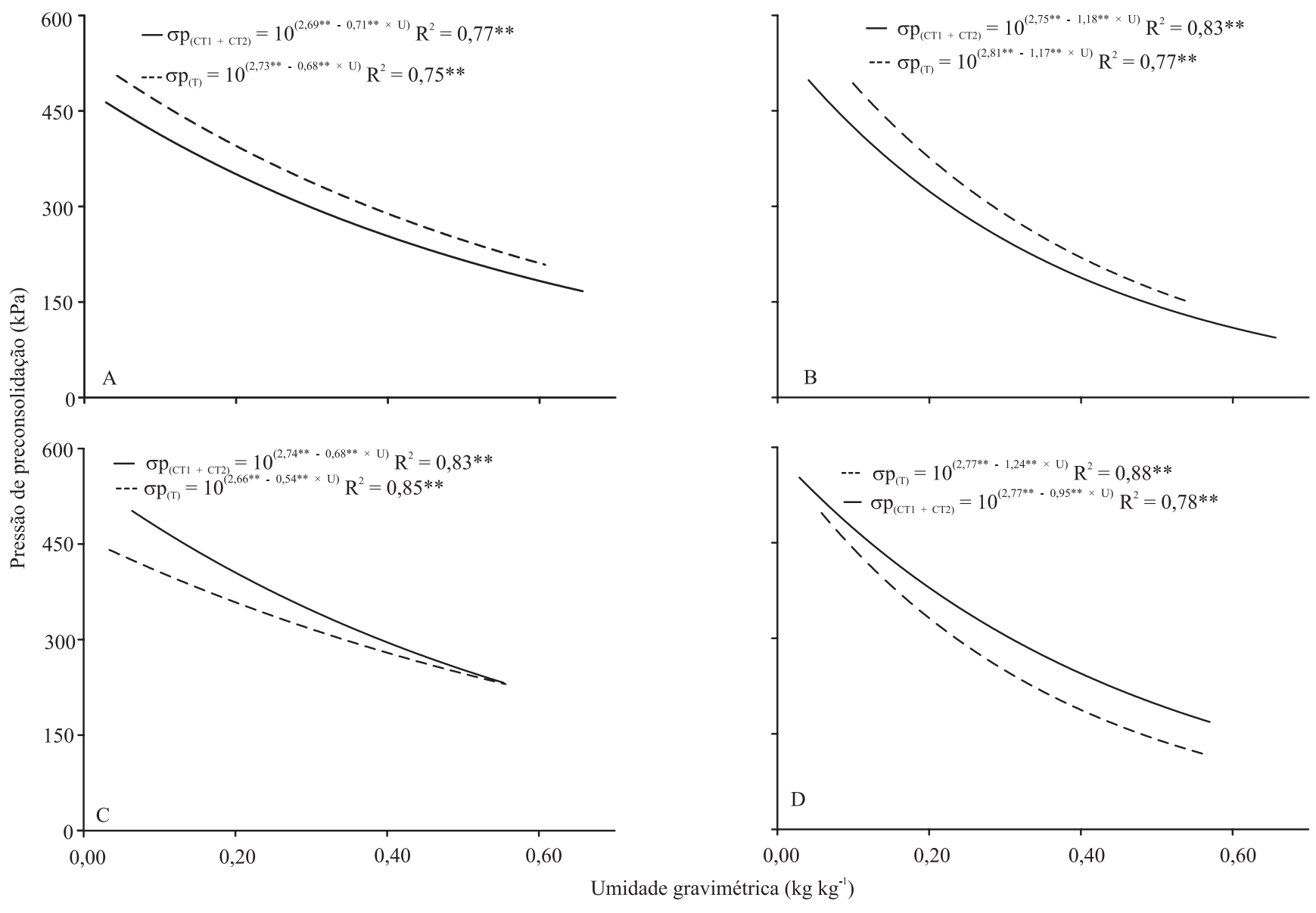

Figura 2. Modelos de capacidade de suporte de carga do solo, para os sistemas de manejo com controle de tráfego (CT1+CT2, $\mathrm{n}=40$ ) e sem controle de tráfego $(\mathrm{T}, \mathrm{n}=20)$, determinados nas linhas do plantio (LP) e de rodado (LR), nas camadas de 0,00-0,10 e 0,20-0,30 m, em A, LP 0,010-0,10 m; B, LP 0,20-0,30 m; C, LR 0,00-0,10 m ; D, LR 0,20-0,30 m. бp, pressão de preconsolidação. 
da compactação do solo próximo à soqueira, o que permitiu a maior produtividade. Contudo, Roque et al. (2010) não constataram diferenças na produtividade da cana-de-açúcar após dois anos de emprego de sistemas de manejo com e sem controle de tráfego.

Foi observada diminuição da densidade do sistema radicular, no sentido da linha de plantio para a linha de rodado, nos três sistemas de manejo avaliados (Figura 3). A menor densidade radicular na linha de rodado está relacionada, principalmente, a limitações físicas do solo causadas pelo tráfego de máquinas. A maior compactação do solo resulta em menor densidade radicular e reduz o volume de solo utilizado, o que pode causar perda de produtividade, principalmente em culturas na condição de sequeiro (Braunack et al.,
2006; Collares et al., 2008). Vasconcelos et al. (2003) observaram redução de $26 \%$ na densidade radicular da cana-de-açúcar, na camada de 0,00-0,20 m, em colheita mecânica comparada à colheita manual. Faroni \& Trivelin (2006) observaram redução de 91\% na concentração do sistema radicular na linha do rodado, em comparação à soqueira, na camada de $0,00-0,20 \mathrm{~m}$, no final do ciclo da cultura; o resultado desses autores é corroborado pelo presente trabalho.

O maior desenvolvimento do sistema radicular foi observado na camada superficial do solo $(0,00-0,10 \mathrm{~m})$ e diminuiu com o aumento na profundidade (Figura 3 ). De acordo com Vasconcelos et al. (2003), 55,4\% da densidade radicular da cana colhida mecanicamente está concentrada na camada de $0,00-0,20 \mathrm{~m}$. Faroni

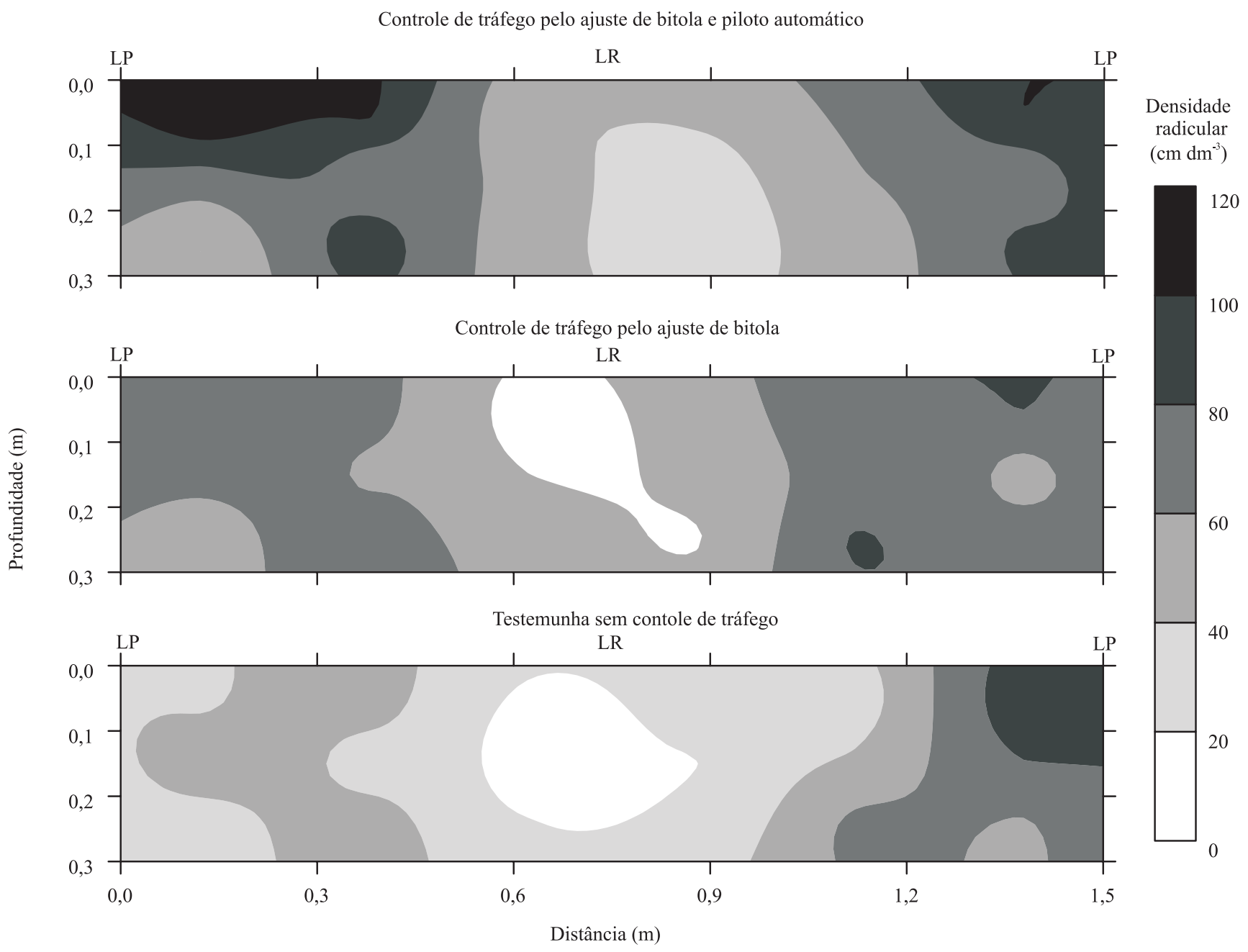

Figura 3. Densidade radicular da cana-de-açúcar, na camada de 0,00-0,30 m, nos seguintes sistemas de manejo com controle de tráfego por meio do ajuste da bitola e uso de piloto automático, e por meio do ajuste da bitola apenas; e testemunha sem controle de tráfego. LP, linha de plantio; LR, linha de rodado. 
\& Trivelin (2006) observaram que $80 \%$ da massa de matéria seca de raízes estava localizada nessa mesma camada.

O ajuste da bitola e o uso do piloto automático podem promover a preservação da qualidade física do solo na linha de plantio, pois se observou que a área de soqueira apresentou maior volume de poros em consequência da menor compactação.

\section{Conclusões}

1. Os sistemas de manejo da cana-de-açúcar com controle de tráfego agrícola propiciam maior capacidade de suporte de carga, na linha de rodado, e possibilitam o tráfego de máquinas agrícolas em solos com maior teor de umidade, devido aos maiores valores de pressão de preconsolidação.

2. O ajuste da bitola do conjunto trator-transbordo para $3,0 \mathrm{~m}$ e o uso de piloto automático contribuem para a preservação da qualidade estrutural do solo, na região da soqueira, e para o maior desenvolvimento do sistema radicular da cana-de-açúcar.

\section{Agradecimentos}

Ao Conselho Nacional de Desenvolvimento Científico e Tecnológico, por concessão de bolsa; à Fundação de Amparo à Pesquisa do Estado de São Paulo, pelo financiamento do projeto; e à Usina São Martinho, por disponibilizar a área de estudo.

\section{Referências}

BOWLES, J.E. Engineering properties of soils and their measurements. $3^{\text {rd }}$ ed. Auckland: McGraw-Hill, 1986. 218p.

BRAUNACK, M.V.; ARVIDSSON, J.; HAKANSSON, I. Effect of harvest traffic position on soil conditions, and sugarcane (Saccharum officinarum) response to environmental conditions in Queensland, Australia. Soil and Tillage Research, v.89, p.103-121, 2006.

BRAUNACK, M.V.; MCGARRY, D. Traffic control and tillage strategies for harvesting and planting of sugarcane (Saccharum officinarum) in Australia. Soil and Tillage Research, v.89, p.86-102, 2006.

CLAESSEN, M.E.C. (Org.). Manual de métodos de análise de solo. 2.ed.rev. atual. Rio de Janeiro: Embrapa-CNPS, 1997. 212p. (Embrapa-CNPS. Documentos, 1).

COLLARES, J.L.; REINERT, D.J.; REICHERT, J.M.; KAISER, D.R. Compactação de um Latossolo induzida pelo tráfego de máquinas e sua relação com o crescimento e produtividade de feijão e trigo. Revista Brasileira de Ciência do Solo, v.32, p.933-942, 2008.

DEBIASI, H.; LEVIEN, R.; TREIN, C.R.; CONTE, O.; MAZURANA, M. Capacidade de suporte e compressibilidade de um Argissolo, influenciadas pelo tráfego e por plantas de cobertura de inverno. Revista Brasileira de Ciência do Solo, v.32, p.2629-2637, 2008.

DIAS JÚNIOR, M. de S. Compression of three soils under long-term tillage and wheel traffic. 1994. 114p. Tesis (Doctor) - Michigan State University, East Lansing.

FARONI, C.E.; TRIVELIN, P.C.O. Quantificação de raízes metabolicamente ativas de cana-de-açúcar. Pesquisa Agropecuária Brasileira, v.41, p.1007-1013, 2006.

IMHOFF, S.; SILVA, A.P. da; FALLOW, D. Susceptibility to compaction, load support capacity, and soil compressibility of Hapludox. Soil Science Society of America Journal, v.68, p.17-24, 2004.

JORGE, L.A.C.; CRESTANA, S. SIARCS: Sistema Integrado para Análise de Raízes e Cobertura do Solo. Versão 3.0. São Carlos: Embrapa-CNPDIA, 1996.

KAISER, D.R.; REINERT, D.J.; REICHERT, J.M.; COLLARES, G.L.; KUNZ, M. Intervalo hídrico ótimo no perfil explorado pelas raízes de feijoeiro em um Latossolo sob diferentes níveis de compactação. Revista Brasileira de Ciência do Solo, v.33, p.845-855, 2009.

KELLER, T.; ARVIDSSON, J. Technical solutions to reduce the risk of subsoil compaction: effects of dual wheels, tandem wheels and tyre inflation pressure on stress propagation in soil. Soil and Tillage Research, v.79, p.191-205, 2004.

KELLER, T.; ARVIDSSON, J.; DAWIDOWSKI, J.B.; KOOLEN, A.J. Soil precompression stress II. A comparison of different compaction tests and stress-displacement behavior of the soil during wheeling. Soil and Tillage Research, v.77, p.97-108, 2004.

KELLER, T.; LAMANDÉ, M. Challenges in the development of analytical soil compaction models. Soil and Tillage Research, v.111, p.54-64, 2010.

KINGWELL, R.; FUCHSBICHLER, A. The whole-farm benefits of controlled traffic farming: an Australian appraisal. Agricultural Systems, v.104, p.513-521, 2011.

LIMA, C.L.R. de; SILVA, A.P. da; IMHOFF, S.C.; LEÃO, T.P. Estimativa da capacidade de suporte de carga do solo a partir da avaliação da resistência à penetração. Revista Brasileira de Ciência do Solo, v.30, p.217-223, 2006.

PACHECO, E.P.; CANTALICE, J.R.B. Compressibilidade, resistência à penetração e intervalo hídrico ótimo de um Argissolo Amarelo cultivado com cana-de-açúcar nos Tabuleiros Costeiros de Alagoas. Revista Brasileira de Ciência do Solo, v.35, p.403-415, 2011.

ROQUE, A.A. de O.; SOUZA, Z.M. de; BARBOSA, R.S.; SOUZA, G.S. de. Controle de tráfego agrícola e atributos físicos do solo em área cultivada com cana-de-açúcar. Pesquisa Agropecuária Brasileira, v.45, p.744-750, 2010. 
SAFFIH-HDADI, K.; DÉFOSSEZ, P.; RICHARD, G.; CUI, Y.J.; TANG, A.M.; CHAPLAIN, V. A method for predicting soil susceptibility to the compaction of surface layers as a function of water content and bulk density. Soil and Tillage Research, v.105, p.96-103, 2009.

SANTOS, H.G. dos; JACOMINE, P.K.T.; ANJOS, L.H.C. dos; OLIVEIRA, V.A. de; OLIVEIRA, J.B. de; COELHO, M.R.; LUMBRERAS, J.F.; CUNHA, T.J.F. (Ed.). Sistema brasileiro de classificação de solos. 2.ed. Rio de Janeiro: Embrapa Solos, 2006. 306p.

SILVA, R.B. da; LANÇAS, K.P.; CARDOSO, V.M.F.; MIRANDA, E.E.V. de. Atributos físicos, mecânicos e dinâmicos do solo como indicadores do impacto do cultivo e do tráfego em perímetros irrigados. Irriga, v.11, p.384-401, 2006.

SILVA, R.B. da; LANÇAS, K.P.; MIRANDA, E.E.V.; SILVA, F.A.M.; BAIO, F.H.R. Estimation and evaluation of dynamic properties as indicators of changes on soil structure in sugarcane fields of Sao Paulo State - Brazil. Soil and Tillage Research, v.103, p.265-270, 2009.
SNEDECOR, G.W.; COCHRAN, W.G. Statistical methods. $8^{\text {th }}$ ed. Ames: Iowa State University Press, 1989. 503p.

SOWERS, G.F. Consistency. In: BLACK, C.A. (Ed.). Methods of soil analysis: physical and mineralogical properties, including statistics of measurements and sampling. Madison: American Society of Agronomy, 1965. p.391-399.

SUZUKI, L.E.A.S.; REINERT, D.J.; REICHERT, J.M.; LIMA, C.L.R. Estimativa da susceptibilidade à compactação e do suporte de carga do solo com base em propriedades físicas de solos do Rio Grande do Sul. Revista Brasileira de Ciência do Solo, v.32, p.963-973, 2008.

VASCONCELOS, A.C.M.; CASAGRANDE, A.A.; PERECIN, D.; JORGE, L.A.C.; LANDELL, M.G.A. Avaliação do sistema radicular da cana-de-açúcar por diferentes métodos. Revista Brasileira de Ciência do Solo, v.27, p.849-858, 2003.

VERMEULEN, G.D.; MOSQUERA, J. Soil, crop and emission responses to seasonal-controlled traffic in organic vegetable farming on loam soil. Soil and Tillage Research, v.102, p.126-134, 2009.

Recebido em 21 de dezembro de 2011 e aprovado em 23 de março de 2012 